\title{
Effect Algebras of Positive Self-adjoint Operators Densely Defined on Hilbert Spaces
}

\author{
Z. Riečanová
}

\begin{abstract}
We show that (generalized) effect algebras may be suitable very simple and natural algebraic structures for sets of (unbounded) positive self-adjoint linear operators densely defined on an infinite-dimensional complex Hilbert space. In these cases the effect algebraic operation, as a total or partially defined binary operation, coincides with the usual addition of operators in Hilbert spaces.
\end{abstract}

Keywords: quantum structures, (generalized) effect algebra, Hilbert space, (unbounded) positive linear operator.

\section{Introduction}

For any linear operator $A$ densely defined on a Hilbert space $\mathcal{H}$ one can define its adjoint operator $A^{*}$. If $A^{*}$ coincides with $A$ then operator $A$ is called selfadjoint. Self-adjoint (unbounded) linear operators on infinite-dimensional complex Hilbert spaces have importance in quantum mechanics, since they represent physical observables, e.g. the position or momentum of an elementary particle. Differential operators form a class of unbounded operators. The Laplace operator is an example of an unbounded positive linear operator.

The algebraic structures of sets of such operators distinguish from classical boolean logics. This follows from the fact that e.g., the distributive law fails due to the noncompatibility of some pairs of operators. For instance, position $x$ and momentum $p$ of an elementary particle cannot be measurable simultaneously with arbitrarily prescribed accuracy, hence $x$ and $p$ are noncompatible. Non-classical logic for calculus of propositions of quantum mechanical system started in 1936 by Birkhoff and von Neuman, (see [2]). Effect algebras were introduced in 1994 in [5]. A survey of algebras of unbounded operators can be found in [1].

The aim of this paper is to show that (generalized) effect algebras may be suitable, very simple and natural algebraic structures for sets of linear operators (including unbounded ones) densely defined on an infinite-dimensional complex Hilbert space, at which the effect algebraic operation coincides with the usual sum of operators.

More details on linear operators on Hilbert spaces can be found, e.g., in [3] and about effect algebras in [4].

\section{Basic definitions and some known facts}

In the paper we assume that $\mathcal{H}$ is an infinitedimensional complex Hilbert space, i.e., a linear space with inner product $(\cdot, \cdot)$ which is complete in the induced metric. Conventions differ as to which argument sesquilinear form $(\cdot, \cdot)$ should be linear. Recall that here for any $x, y \in \mathcal{H}$ we have $(x, y) \in \mathbb{C}$ (the set of complex numbers) such that $(x, \alpha y+\beta z)=$ $\alpha(x, y)+\beta \underline{(x, z)}$ for all $\alpha, \beta \in \mathbb{C}$ and $x, y, z \in \mathcal{H}$. Moreover, $\overline{(x, y)}=(y, x)$ and finally $(x, x) \geq 0$ at which $(x, x)=0$ iff $x=0$. The term dimension of $\mathcal{H}$ in the following always means the Hilbertian dimension defined as the cardinality of any orthonormal basis of $\mathcal{H}$ (see [3, p. 44]).

Moreover, we will assume that all considered linear operators $A$ (i.e., linear maps $A: D(A) \rightarrow \mathcal{H}$ ) have a domain $D(A)$ a linear subspace dense in $\mathcal{H}$ with respect to metric topology induced by inner product, so $\overline{D(A)}=\mathcal{H}$. Moreover, our next results will be for positive linear operators $A$ (denoted by $A \geq 0)$, meaning that $(A x, x) \geq 0$ for all $x \in D(A)$, therefore operators $A$ are also symmetric, (for more details see [3]). We will denote the set of all such operators by $\mathcal{V}(\mathcal{H})$.

Recall that $A: D(A) \rightarrow \mathcal{H}$ is called a bounded operator if there exists a real constant $C \geq 0$ such that $\|A x\| \leq C\|x\|$ for all $x \in D(A)$ and hence $A$ is an unbounded operator if to every $C$ there exists $x_{C} \in D(A)$ with $\left\|A x_{C}\right\|>C\left\|x_{C}\right\|$. To every linear operator with $\overline{D(A)}=\mathcal{H}$ there exists the adjoint linear operator $A^{*}$ of $A$ such that $D\left(A^{*}\right)=\{y \in \mathcal{H} \mid$ there exists $y^{*} \in \mathcal{H}$ such that $\left(y^{*}, x\right)=(y, A x)$ for all $x \in D(A)\}$ and $A^{*} y=y^{*}$ for every $y \in D\left(A^{*}\right)$. 
If $A^{*}=A$ then $A$ is called self-adjoint. The set of all positive self-adjoint linear operators densely defined in $\mathcal{H}$ will be denoted by $\mathcal{S}_{p}(\mathcal{H})$. Hence $\mathcal{S}_{p}(\mathcal{H})=\left\{A \in \mathcal{V}(\mathcal{H}) \mid A=A^{*}\right\}$.

A densely defined linear operator $A$ on $\mathcal{H}$ is called symmetric, if $A \subset A^{*}$. Here we write $A \subset B$ iff $D(A) \subseteq D(B)$ and $A x=B x$ for every $x \in D(A)$. The condition $A \subset A^{*}$ is equivalent to $(y, A x)=$ $(A y, x)$ for all $x, y \in D(A)$.

An operator $A: D(A) \rightarrow \mathcal{H}$ is called closed if for every sequence $\left(x_{n}\right)_{n \in \mathbb{N}}, x_{n} \in D(A)$, such that $x_{n} \rightarrow x \in \mathcal{H}$ and $A x_{n} \rightarrow y \in \mathcal{H}$ as $n \rightarrow \infty$ one has $x \in D(A)$ and $A x=y$. Since $A \in \mathcal{V}(\mathcal{H})$ is positive and hence also symmetric (see [3], p. 142) there exists a closed operator $\bar{A}$ such that $A \subset \bar{A}$ and $\bar{A} \subset B$ for every closed operator $B \supset A$. Moreover $\bar{A}$ is symmetric and it is called the closure of $A$. A symmetric operator is called essentially self-adjoint if $(\bar{A})^{*}=\bar{A}$ and then $\bar{A}$ is a unique self-adjoint extension of $A[3$, p. 96].

We shall show in Section 3 that, under a partially defined usual sum of linear operators, sets $\mathcal{V}(\mathcal{H})$ and $\mathcal{S}_{p}(\mathcal{H})$ form quantum structures called (generalized) effect algebras (see also [9]). Now we recall their definitions.

Definition 2.1 (Foulis and Bennett, 1994) A partial algebra $(E ; \oplus, 0,1)$ is called an effect algebra if 0,1 are two distinguished elements and $\oplus$ is a partially defined binary operation on $E$ which satisfies the following conditions for any $x, y, z \in E$ :

(E1) $x \oplus y=y \oplus x$ if $x \oplus y$ is defined,

(E2) $(x \oplus y) \oplus z=x \oplus(y \oplus z)$ if one side is defined,

(E3) for every $x \in E$ there exists a unique $y \in E$ such that $x \oplus y=1$ (we put $x^{\prime}=y$ ),

(E4) If $1 \oplus x$ is defined then $x=0$.

We often denote the effect algebra $(E ; \oplus, 0,1)$ briefly by $E$. On every effect algebra $E$ the partial order $\leq$ and partial binary operation $\ominus$ can be introduced as follows:

$x \leq y$ and $y \ominus x=z$ iff $x \oplus z$ is defined and $x \oplus z=y$.

If $E$ with the defined partial order is a lattice (a complete lattice) then $(E ; \oplus, 0,1)$ is called a lattice effect algebra (a complete lattice effect algebra).

Generalizations of effect algebras (i.e., without a top element 1) have been studied by Kôpka and Chovanec (1994) (difference posets), Foulis and Bennett (1994) (cones), Kalmbach and Riečanová (1994) (abelian $R I$-posets and abelian $R I$ semigroups) and Hedlíková and Pulmannová (1996) (generalized $D$ posets and cancellative positive partial abelian semigroups). It can be shown that all of the above mentioned generalizations of effect algebras are mutually equivalent and extend similar previous results for generalized Boolean algebras and orthomodular lattices and posets.

\section{Definition 2.2}

(1) A generalized effect algebra $(E, \oplus, 0)$ is a set $E$ with element $0 \in E$ and partial binary operation $\oplus$ satisfying for any $x, y, z \in E$ conditions

(GE1) $x \oplus y=y \oplus x$ if one side is defined,

(GE2) $(x \oplus y) \oplus z=x \oplus(y \oplus z)$ if one side is defined,

(GE3) if $x \oplus y=x \oplus z$ then $y=z$,

(GE4) if $x \oplus y=0$ then $x=y=0$,

(GE5) $x \oplus 0=x$ for all $x \in E$.

(2) A binary relation $\leq$ (being a partial order) on $E$ can be defined by

$$
x \leq y \text { iff for some } z \in E, x \oplus z=y \text {. }
$$

(3) $Q \subseteq E$ is called a sub-generalized effect algebra (sub-effect algebra) of the generalized effect algebra $E$ (effect algebra $E$ ) iff it has the following property. If at least two of elements $x, y, z \in E$ with $x \oplus y=z$ are in $Q$ then all $x, y, z$ are in $Q$.

Note that a sub-generalized effect algebra (sub-effect algebra) $Q \subset E$ is a (generalized) effect algebra in its own right.

\section{Generalized effect algebras of positive operators on a Hilbert space and their sub-generalized effect algebras}

In [9] the following theorem on positive linear operators with common domain was proved:

Theorem 3.1 $[9$, Theorem 3.1] Let $\mathcal{H}$ be a complex Hilbert space and let $D \subseteq \mathcal{H}$ be a linear subspace dense in $\mathcal{H}$ (i.e., $\bar{D}=\mathcal{H})$. Let

$$
\begin{aligned}
\mathcal{G}_{D}(\mathcal{H})= & \{A: D \rightarrow \mathcal{H} \mid A \text { is a positive } \\
& \text { linear operator defined on } D\} .
\end{aligned}
$$

Then $\left(\mathcal{G}_{D}(\mathcal{H}) ; \oplus, 0\right)$ is a generalized effect algebra where 0 is the null operator and $\oplus$ is the usual sum of operators defined on D. In this case $\oplus$ is a total operation.

If $D=\mathcal{H}$ in Theorem 3.1 then $\mathcal{G}_{D}(\mathcal{H})$ is a generalized effect algebra of all bounded positive linear operators acting in $\mathcal{H}$ with usual addition as effect algebraic operation $\oplus$. Hence in the case $D=\mathcal{H}$ all operators in $\mathcal{G}_{D}(\mathcal{H})$ are self-adjoint.

On the other hand, if $D \neq \mathcal{H}$ then every bounded operator in $\mathcal{G}_{D}(\mathcal{H})$ is a restriction $A \mid D$ of a bounded operator $A$ with $D(A)=\mathcal{H}$. Thus, in this case, $A=A^{*}=(A \mid D)^{*} \neq A \mid D$. It follows that every 
self-adjoint operator in $\mathcal{G}_{D}(\mathcal{H})$ for $D \neq \mathcal{H}$ is necessarily unbounded. Nevertheless, it is well known (see, e.g. [10]) that every densely defined positive operator $A$ has a positive self-adjoint extension $\hat{A}$ called Friedrichs' extension. Moreover, $\hat{A}$ extends all symmetric extension $A^{\prime}$ of $A$. Thus if $A^{\prime}$ is selfadjoint then $A^{\prime}=\hat{A}$. But in general $D(A) \neq D(\hat{A})$, hence $\hat{A} \notin \mathcal{G}_{D}(\mathcal{H})$. Clearly, for domains $D_{1} \neq D_{2}$, $\mathcal{G}_{D_{1}}(\mathcal{H}) \cap \mathcal{G}_{D_{2}}(\mathcal{H})=\emptyset$. However it is well-known that bounded linear operators have unique extensions to the whole space $\mathcal{H}$. Theorem 3.1 remains true if we substitute $\mathcal{G}_{D}(\mathcal{H})$ by

$$
\begin{aligned}
\widetilde{\mathcal{G}}_{D}(\mathcal{H})= & \{A: D(A) \rightarrow \mathcal{H} \mid A \text { is positive } \\
& \text { linear operator with } \\
& D(A)=D \text { if } A \text { is unbounded, } \\
& D(A)=\mathcal{H} \text { if it is bounded }\}
\end{aligned}
$$

Then for $D_{1} \neq D_{2}$ we obtain $\widetilde{\mathcal{G}}_{D_{1}}(\mathcal{H}) \cap \widetilde{\mathcal{G}}_{D_{2}}(\mathcal{H})=$ $\mathcal{B}^{+}(\mathcal{H})$ where $\mathcal{B}^{+}(\mathcal{H})$ is the set of all bounded positive linear operators $A$ with $D(A)=\mathcal{H}$.

Theorem 3.2 $[9$, Theorem 3.5] Let $\mathcal{H}$ be an infinite-dimensional complex Hilbert space. Let

$$
\begin{aligned}
\mathcal{V}(\mathcal{H})= & \{A: D(A) \rightarrow \mathcal{H} \mid A \geq 0 \text { with } \\
& \overline{D(A)}=\mathcal{H} \text { and } \\
& D(A)=\mathcal{H} \text { if } A \text { is bounded }\}
\end{aligned}
$$

Let $\oplus$ be a partial binary operation on $\mathcal{V}(\mathcal{H})$ defined by $A \oplus B=A+B$ with $D(A \oplus B)=\mathcal{H}$ for any bounded $A, B \in \mathcal{V}(\mathcal{H})$ and $A \oplus B=B \oplus A=A+\left.B\right|_{D(A)}$ with $D(A \oplus B)=D(A)$ if $A$ is unbounded and $B$ is bounded.

Then $(\mathcal{V}(\mathcal{H}) ; \oplus, 0)$ is a generalized effect algebra. Moreover, $\mathcal{B}^{+}(\mathcal{H})$ is a sub-generalized effect algebra of $\mathcal{V}(\mathcal{H})$ with respect to inherited $\oplus$-operation, which is defined for every pair $A, B \in \mathcal{B}^{+}(\mathcal{H})$.

Now we are going to show that

$$
\mathcal{S}_{p}(\mathcal{H})=\left\{A \in \mathcal{V}(\mathcal{H}) \mid A=A^{*}\right\}
$$

is a sub-generalized effect algebra of $\mathcal{V}(\mathcal{H})$, hence it is a generalized effect algebra. Moreover, we show that

$$
\begin{aligned}
& \mathcal{S}_{p}(\mathcal{H})=\mathcal{F}(\mathcal{H})=\{\hat{A} \mid A \in \mathcal{V}(\mathcal{H}), \hat{A} \\
& \text { is a Friedrichs positive } \\
&\text { self-adjoint extension of } A\} .
\end{aligned}
$$

Lemma 3.3 Under the assumptions of Theorem 3.2, for every $A \in \mathcal{V}(\mathcal{H})$ :

(i) $\bar{A}$ and $A^{*}$ exist, $\hat{A}$ is closed and $A \subset \bar{A} \subset \hat{A}=$ $(\hat{A})^{*} \subset(\bar{A})^{*}=A^{*}$,

(ii) $\hat{A}=\bar{A}$ iff $A$ is essentially self-adjoint,

(iii) $S_{p}(\mathcal{H})=\mathcal{F}(\mathcal{H})$.

Proof. (i) Let $A \in \mathcal{V}(\mathcal{H})$. Since $\overline{D(A)}=\mathcal{H}$, the adjoint $A^{*}$ of $A$ exists [3, p. 93]. Further the assumption that $A \geq 0$ implies that $A$ is symmetric (see [3, p. 142]) and there exists the so-called Friedrichs positive self-adjoint extension $\hat{A}$ of $A$ (see, e.g. [3] or [10]), hence $A \subset \hat{A}=(\hat{A})^{*} \subset A^{*}$. It follows that $\mathcal{H}=\overline{D(A)}=\overline{D(\hat{A})}=\overline{D\left(A^{*}\right)}$, which gives that $A^{*}$ and $(\hat{A})^{*}$ are closed (see $[3$, p. 95$]$ ). As $\hat{A}=(\hat{A})^{*}$, we obtain that $\hat{A}$ is closed. Moreover, since $A$ is symmetric, its closure $\bar{A}$ is also symmetric (see [3, p. 96]). Thus we obtain $A \subset \bar{A} \subset \hat{A}=(\hat{A})^{*} \subset(\bar{A})^{*} \subset A^{*}$. Further $A^{* *}=\bar{A}$ and $(\bar{A})^{*}=A^{*}$ (see $[3$, p. 96$]$ ).

(ii) If $A$ is essentially self-adjoint then $(\bar{A})^{*}=\bar{A}$ implies $\hat{A}=\bar{A}$. Conversely, if $\bar{A}=\hat{A}$ then $\bar{A}=\hat{A}=$ $(\hat{A})^{*}=(\bar{A})^{*}$.

(iii) If $A \in \mathcal{S}_{p}(\mathcal{H})$ then $A=A^{*}$, hence, by (i), $A=\hat{A} \in \mathcal{V}(\mathcal{H})$. Conversely, if $A \in \mathcal{V}(\mathcal{H})$ then $A$ is self-adjoint, hence $A \in \mathcal{S}_{p}(\mathcal{H})$.

Theorem 3.4 Under the assumption of Theorem 3.2 let $\mathcal{S}_{p}(\mathcal{H})=\left\{A \in \mathcal{V}(\mathcal{H}) \mid A=A^{*}\right\}$ and let $\oplus_{S}=\oplus / \mathcal{S}_{p}(\mathcal{H})$ be the restriction of $\oplus$-operation defined on $\mathcal{V}(\mathcal{H})$ to the set $\mathcal{S}_{p}(\mathcal{H})$. Then $\left(\mathcal{S}_{p}(\mathcal{H}) ; \oplus_{S}, 0\right)$ is a sub-generalized effect algebra of $(\mathcal{V}(\mathcal{H}) ; \oplus, 0)$.

Proof. We have to show that if $A, B, C \in \mathcal{V}(\mathcal{H})$ with $A \oplus B=C$ and out of $A, B, C$ at least two are in $\mathcal{S}_{p}(\mathcal{H})$ then $A, B, C \in \mathcal{S}_{p}(\mathcal{H})$.

(i) Assume first that $A, B \in \mathcal{S}_{p}(\mathcal{H})$. If $A, B$ are bounded then $C=A \oplus B$ is again bounded and $D(A)=D(B)=D(C)=\mathcal{H}$, hence $C \in \mathcal{S}_{p}(\mathcal{H})$. Further, if $A$ is unbounded and $B$ is bounded then $C=A+B \mid D(A)$ and $D(C)=D(A)$. Moreover, $A, B \in \mathcal{S}_{p}(\mathcal{H})$ implies that $A=A^{*}$, hence $D(A)=$ $D\left(A^{*}\right)$ and $B=B^{*} \subset(B \mid D(A))^{*}$, which gives $B=$ $(B \mid D(A))^{*}$ on $\mathcal{H}$. It follows, as $B \mid D(A)$ is bounded, that $(A \oplus B)^{*}=(A+B \mid D(A))^{*}=A^{*}+(B \mid D(A))^{*}=$ $A^{*}+B=A^{*}+B\left|D\left(A^{*}\right)=A+B\right| D(A)=A \oplus B$. Again $C \in \mathcal{S}_{p}(\mathcal{H})$.

(ii) Assume now that $A, C \in \mathcal{S}_{p}(\mathcal{H})$. Then if $C$ is bounded then $D(C)=\mathcal{H}$ and then $A, B$ are bounded, hence $A, B \in \mathcal{S}_{p}(\mathcal{H})$. If $C$ and $A$ are unbounded then $B$ is bounded (since otherwise $A \oplus B$ is not defined) and again $B \in \mathcal{S}_{p}(\mathcal{H})$. Finally, if $C$ is unbounded and $A$ is bounded then $B$ is unbounded and $C=A \mid D(B)+B$. It follows that $D(C)=D(B)$. Moreover, $C^{*}=(A \mid D(B))^{*}+B^{*}=A+B^{*}$, hence $D\left(C^{*}\right)=D\left(B^{*}\right)$. Now, the assumption that $C$ is self-adjoint implies $D(C)=D\left(C^{*}\right)$ which gives $D\left(B^{*}\right)=D(B)$, hence $B \in \mathcal{S}_{p}(\mathcal{H})$.

In Theorem 3.4 we may substitute $\mathcal{S}_{p}(\mathcal{H})$ by $\mathcal{F}(\mathcal{H})$. Hence $\mathcal{F}(\mathcal{H})$ is a generalized effect algebra, more precisely:

Corollary 3.5 Let $\mathcal{H}$ be an infinite-dimensional complex Hilbert space. Let $\mathcal{F}(\mathcal{H})$ be the set of all Friedrichs positive self-adjoint extensions of all positive densely defined linear operators in $\mathcal{H}$ with $D(A)=\mathcal{H}$ if $A$ is bounded. Let $\oplus$ be a partial binary operation defined for $A, B \in \mathcal{F}(\mathcal{H})$ iff out 
of operators $A, B$ at least one is bounded and then $A \oplus B=A+B$ is the usual sum of operators in $\mathcal{H}$. Then $(\mathcal{F}(\mathcal{H}) ; \oplus, 0)$ is a generalized effect algebra.

Assume that $(E ; \oplus, 0)$ is a generalized effect algebra. Then (see, e.g., [11]) for any fixed $q \in E, q \neq 0$ the interval

$[0, q]_{E}=\{x \in E \mid$ there exists $y \in E$ with $x \oplus y=q\}$

is an effect algebra $\left([0, q]_{E} ; \oplus_{q}, 0, q\right)$ with unit $q$ and with the partial operation $\oplus_{q}$ defined for $x, y \in[0, q]_{E}$ by

$$
\begin{aligned}
& x \oplus_{q} y \text { exists and } x \oplus_{q} y=x \oplus y \\
& \text { iff } x \oplus y \in[0, q]_{E} \text { exists in } E .
\end{aligned}
$$

We have shown that $\mathcal{V}(\mathcal{H})$ and $\mathcal{S}_{p}(\mathcal{H})$ are generalized effect algebras under the partial operations $\oplus$ and $\oplus_{S}$, respectively. Moreover, $A \oplus B$ (for $A, B \in \mathcal{V}(\mathcal{H})$ ) and $A \oplus_{S} B$ (for $A, B \in \mathcal{S}_{p}(\mathcal{H})$ ) coincide with the usual sum of operators $A, B$ when at least one of them is bounded. If both $A, B$ are unbounded then $A \oplus B, A \oplus_{S} B$, respectively, are not defined. Since for any fixed $Q \in \mathcal{S}_{p}(\mathcal{H}), Q \neq 0$, it holds $[0, Q]_{\mathcal{S}_{p}(\mathcal{H})}=$ $[0, Q]_{\mathcal{V}(\mathcal{H})} \cap \mathcal{S}_{p}(\mathcal{H})$, we obtain the following effect algebras of positive self-adjoint operators:

Theorem 3.6 Let $Q \in \mathcal{S}_{p}(\mathcal{H}), Q \neq 0$ be fixed. Then $\left([0, Q]_{\mathcal{S}_{p}(\mathcal{H})} ; \oplus_{Q}, 0, Q\right)$ is an effect algebra (with unit $Q)$ of positive self-adjoint operators densely defined in $\mathcal{H}$ under the $\oplus_{Q}$ defined for $A, B \in[0, Q]_{\mathcal{S}_{p}(\mathcal{H})}$ by: $A \oplus_{Q} B$ exists and $A \oplus_{Q} B=A+B$ (the usual sum of $A, B$ in $\mathcal{H}$ ) iff at least one out of operators $A, B$ is bounded and $A+B \in[0, Q]_{\mathcal{V}(\mathcal{H})}$.

Note that if we substitute $\mathcal{S}_{p}(\mathcal{H})$ in the preceding theorem by $\mathcal{V}(\mathcal{H})$ then for every fixed $Q \in \mathcal{V}(\mathcal{H})$ we have $[0, Q]_{\mathcal{V}(\mathcal{H})}=\{A \in \mathcal{V}(\mathcal{H}) \mid$ there exists $C \in \mathcal{V}(\mathcal{H})$ such that out of $A, C$ at least one is bounded and $A+C=Q\}$. Then $\left([0, Q]_{\mathcal{V}(\mathcal{H})} ; \oplus_{Q}, 0, Q\right)$ is an effect algebra with unit $Q$ and a partial binary operation $\oplus_{Q}$ defined in Theorem 3.6.

Remark 3.7 (i) If $Q \in \mathcal{S}_{p}(\mathcal{H})$ is a bounded operator then $[0, Q]_{\mathcal{S}_{p}(\mathcal{H})}=[0, Q]_{\mathcal{V}(\mathcal{H})}$ and it is an effect algebra of all bounded self-adjoint positive operators between 0 and $Q$ (with domain $\mathcal{H}$ ). Moreover, $\oplus_{Q}$ coincides with the usual sum of operators if $A \oplus_{Q} B$ exists in $[0, Q]_{\mathcal{S}_{p}(\mathcal{H})}$.

(ii) It follows from (i) that if $Q=I$ (the identity operator with domain $\mathcal{H})$ then $[0, Q]_{\mathcal{S}_{p}(\mathcal{H})}=$ $[0, Q]_{\mathcal{V}(\mathcal{H})}=\mathcal{E}(\mathcal{H})$ is the Hilbert space effect algebra of all self-adjoint operators between 0 and the identity operator $I$ (see [5]).

(iii) If $Q \in \mathcal{S}_{p}(\mathcal{H})$ is an unbounded operator with $\overline{D(Q)}=\mathcal{H}$ then every unbounded operator $A \in$ $[0, Q]_{\mathcal{S}_{p}(\mathcal{H})}$ has $D(A)=D(Q)$, since then there exists a bounded operator $C \in \mathcal{S}_{p}(\mathcal{H})$ (hence $D(C)=\mathcal{H}$ ) such that $A+C=Q$. (iv) If $Q \in \mathcal{S}_{p}(\mathcal{H})$ is an unbounded self-adjoint operator then $(2 Q)^{*}=2 Q^{*}=2 Q \in \mathcal{S}_{p}(\mathcal{H})$. In this case for any operators $A, B \in[0, Q]_{\mathcal{S}_{p}(\mathcal{H})}$ one has $A+B \in \mathcal{S}_{p}(\mathcal{H})$ (the usual sum of operators), even if $A, B$ are unbounded. The last follows from the fact that there are bounded operators $C_{A}, C_{B} \in \mathcal{S}_{p}(\mathcal{H})$ such that $Q=A \oplus C_{A}=B \oplus C_{B}$. Thus $\left(A \oplus C_{A}\right)+$ $\left(B \oplus C_{B}\right)=2 Q$, hence $(A+B)+\left(C_{A}+C_{B}\right)=2 Q$. Here $C_{A}+C_{B} \in \mathcal{S}_{p}(\mathcal{H})$ and because $\mathcal{S}_{p}(\mathcal{H})$ is a generalized effect algebra and also $2 Q \in \mathcal{S}_{p}(\mathcal{H})$ we obtain that $A+B \in \mathcal{S}_{p}(\mathcal{H})$.

(v) It is worth noting that effect algebras are very natural structures as carriers of states (or probability measures) when we handle also noncompatible pairs or unsharp elements.

\section{Acknowledgement}

Supported by VEGA 1/0297/11 grant of the Ministry of Education of the Slovak Republic.

\section{References}

[1] Bagarello, F.: Algebras of unbounded operators and physical applications: A survey, Reviews in Mathematical Physics 19 (2007), 231-271.

[2] Birkhoff, G., von Neumann, J.: The logic of quantum mechanics, Ann. Math. 37 (1936), 823-843.

[3] Blank, J., Exner, P., Havlíček, M.: Hilbert Space Operators in Quantum Physics. (Second edition), Springer, 2008.

[4] Dvurečenskij, A., Pulmannová, S.: New trends in quantum structures. Dodrecht : Kluwer, The Netherlands, 2000.

[5] Foulis, D. J., Bennett, M. K.: Effect algebras and unsharp quantum logics, Found. Phys. 24 (1994), 1331-1352.

[6] Hedlíková, J., Pulmannová, S.: Generalized difference posets and orthoalgebras, Acta Math. Univ. Comenianae LXV (1996), 247-279.

[7] Kalmbach, G., Riečanová, Z.: An axiomatization for abelian relative inverses, Demonstratio Math. 27 (1994), 769-780.

[8] Kôpka, F., Chovanec, F.: D-posets, Math. Slovaca 44 (1994), 21-34.

[9] Polakovič, M., Riečanová, Z.: Generalized effect algebras of positive operators densely defined on Hilbert space, Internat. J. Theor. Phys 50 (2011), 1 167-1 174. 
[10] Reed, M., Simon, B.: Methods of Modern Mathematical Physics II, Fourier Analysis, SelfAdjointness. New York, San Francisco, London : Academic Press, 1975.

[11] Riečanová, Z.: Subalgebras, intervals and central elements of generalized effect algebras, International Journal of Theoretical Physics $\mathbf{3 8}$ (1999), $3209-3220$.
Zdenka Riečanová

E-mail: zdenka.riecanova@stuba.sk Department of Mathematics Faculty of Electrical Engineering and Information Technology STU Ilkovičova 3, SK-81219 Bratislava 